\title{
Acampomelic Campomelic Dysplasia
}

National Cancer Institute

\section{Source}

National Cancer Institute. Acampomelic Campomelic Dysplasia. NCI Thesaurus. Code C120205.

A variant of campomelic dysplasia characterized by the absence of skeletal dysplasia. 